\title{
Estimate of Floor Reaction Force vector using Foot-Pressure Sensor*
}

\author{
Kazuto MIYAWAKI ${ }^{* *}$, Takehiro IWAMI ${ }^{* * *}$, Goro OBINATA ${ }^{* * * *}$ \\ and Yoichi SHIMADA ${ }^{* * * * *}$ \\ ${ }^{* *}$ Akita Prefectural R\&D Center, \\ 4-21 Sanuki Araya-cho, Akita--shi, Akita, 010-1623 Japan \\ E-mail: miyawaki@rdc.pref.akita.jp \\ ***Akita University, \\ 1-1, Tegatagakuen-cho, Akita-shi 010-8502 Japan \\ E-mail: iwami@control.mech.akita-u.ac.jp \\ ****Nagoya University, \\ Furou-cho, Chikusa-ku nagoya-shi Aichi 464-8603 Japan \\ E-mail: obinata@mech.nagoya-u.ac.jp \\ *****Akita University School of Medicine, \\ 1-1, Hondo, Akita-shi, Akita 010-8502 Japan \\ E-mail: yshimada@med.akita-u.ac.jp
}

\begin{abstract}
As people grow older, the ability to walk becomes ever more important to live an independent life. A simple system that is useful for evaluation and analysis of gait is necessary. Our goal is to design a wearable gait analyzer. In this study, we estimated the floor reaction force vector using an ultra-thin pair of wearable sensor pads. The floor reaction force vector, when someone is walking, is in the direction of the body's center of gravity. The gap separating the floor reaction force vector and the body's center of gravity generates a rotation moment, which is what we used to create this equation. Our evaluation method will be useful for future development of sensor systems.
\end{abstract}

Key words: Biomechanics, Bio-Motion, Gait, Wearable, Floor Reaction Force, Joint Moment

\section{Introduction}

The current system used for gait analysis uses a combination of a three-dimensional optical motion capture system that measures the body's locus and a force plate that measures the floor reaction force vector. The system respectively limits the areas of measurement and motion. We propose this system aimed at eventual development of a wearable system. This system will be useful in the future when ascending and descending stairs. Unfortunately, the force plate is large, heavy, and expensive, and a large space is necessary to set it up and to use it. Another problem with using a force plate is that, at times, elderly people as well as people suffering from various disabilities find it difficult to step onto the center of the plate. What we propose in this study is a gait analysis system with a pair of wearable thin sheet foot-pressure sensors instead of the force plate. These foot-pressure sensors necessitate a motion capture system to measure the body gravity. That requirement persists as a weak point. In the future, wearable measuring systems will be important for society ${ }^{(1)}$. For this study we used a pair of foot-pressure sensor pads that measured the center of pressure (COP) as well as the vertical component of floor reaction force vector. Each sensor pad consists of 960 individual sensors that measure the electrical-resistance pressure in a grid like fashion. Although we were able to measure the 
vertical floor reaction force vector when using the foot sensor pads, we were unable to measure the horizontal component of the floor reaction force vector. Therefore, we needed to estimate the horizontal component of the floor reaction force vector. The floor reaction force vector during gait points at the center of gravity $(\mathrm{CG})$, which has a rotational moment when the floor reaction force points away from CG. We used this fact to calculate the horizontal component of the floor reaction force vector.

\section{Evaluation of floor reaction force vector using foot-pressure sensors}

\subsection{Foot Pressure Sensors}

The foot-pressure sensors (F-SCAN) are very thin: $0.1 \mathrm{~mm}$. The sensors can be cut to individual foot sizes, and can then be fitted as shoe insoles, thereby constituting a wearable sensor system that can measure pressure.

\subsection{Estimate of floor reaction force vector using mechanical characteristics}

The vertical component of the floor reaction force can be measured using the foot-pressure sensors. But it can not measure the direction of the force. Furthermore, in this study, we calculate the horizontal component of the floor reaction vector. To obtain information about the direction of force, we must estimate the horizontal component of the floor reaction force vector. The floor reaction force vector during gait points to the $\mathrm{CG}^{(2)}$. Figure 1 shows the normal gait stick picture and the sagittal plane. The vertical axis shows the height above the floor; the horizontal axis shows the gait distance.

Floor reaction force vector

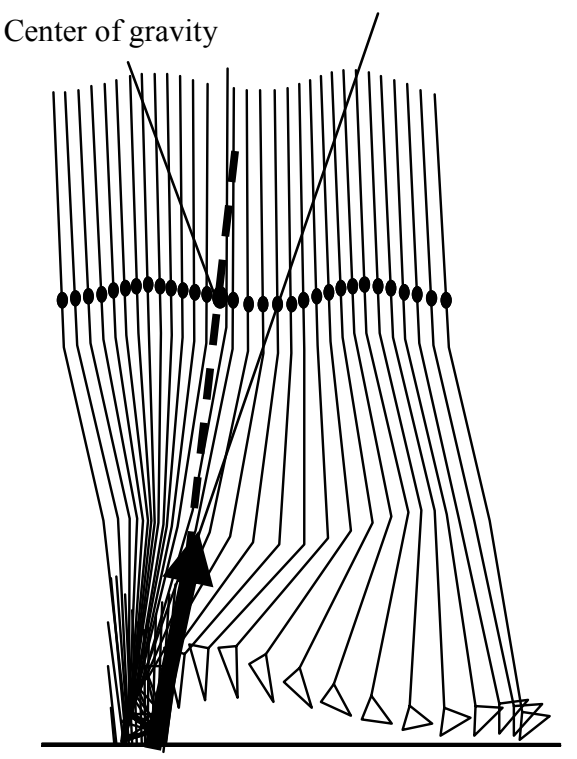

Fg.1 Stick figure

In this study, we apply such a mechanical model as illustrated in Figure 2(a). The only two forces to $\mathrm{CG}$ are applied during gait: gravity and the floor reaction force. Here, the floor reaction force vector during gait is pointing to the CG, the body movement's translation is depicted in Fig. 2(b). The floor reaction force vector during gait is not pointing to the CG, the body moves the rotational moment, as depicted in Fig. 2(c).

When the floor reaction force vector is pointing in the direction of the body's CG, as portrayed in Fig. 2(b), we found the horizontal component floor reaction force shown in eq. (1). It is calculated as the ratio of the body's CG to the acting point of the floor reaction force $(u)$. 


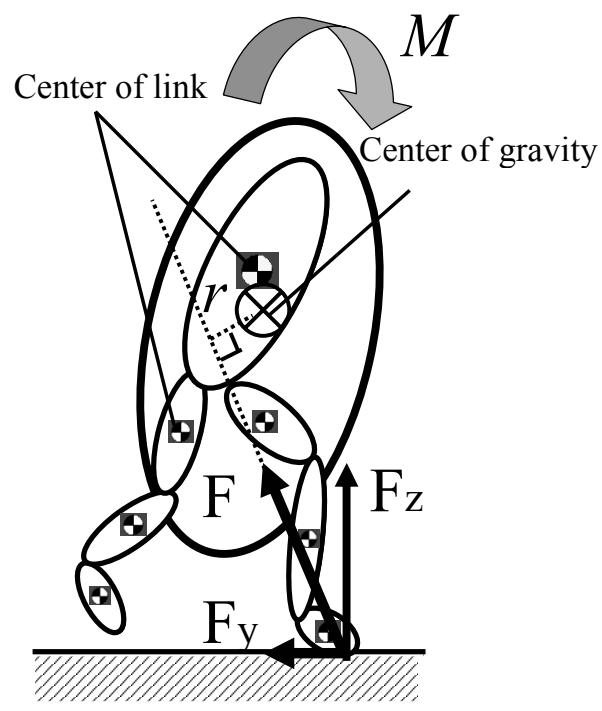

(a) Body Model

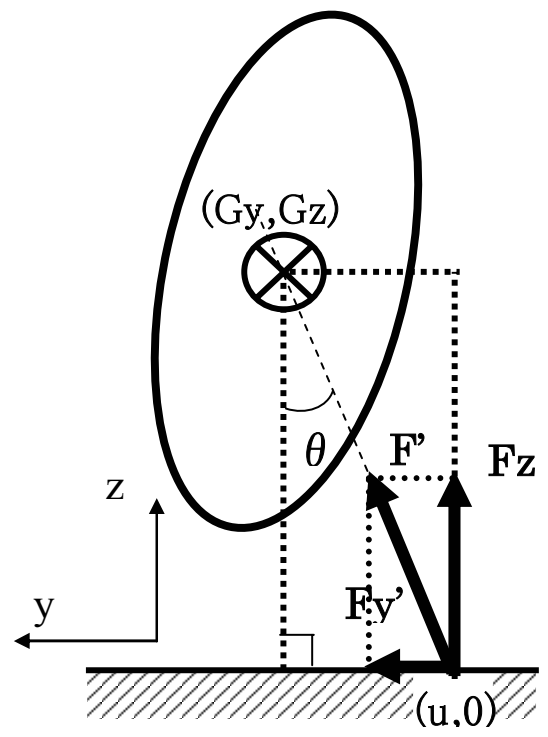

(b) Toward gravity

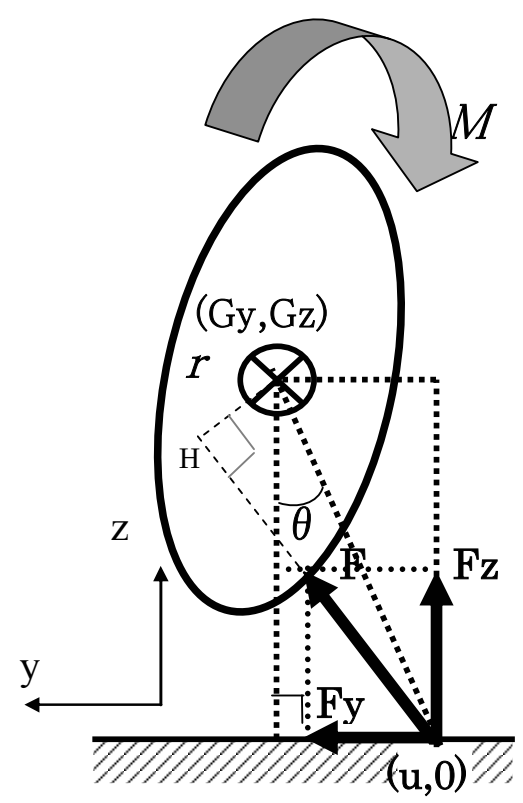

(c) Not toward gravity

Fig. 2 Analysis Mode

When the floor reaction force vector is pointing in the direction of the body's CG, as depicted in Fig. 2(b), we found the horizontal component floor reaction force in eq. (1). It is calculated by the ratio of the body's CG to the acting point of the floor reaction force $(u)$.

$$
F_{y}^{\prime}=\frac{\left(G_{y}-u\right) F_{z}}{G_{z}}
$$

If the floor reaction force vector gets out of position from the CG, as portrayed in Fig. 2(c), we find the horizontal component floor reaction force in eq. (2) because it yields the 
vertical distance ( $r$ ) from the body's CG to the line of the floor reaction force vector. After finding it, we can determine the point of intersection $(\mathrm{H})$.

$$
F_{y}=\frac{\left(G_{y}+r \cdot \cos \theta-u\right) F_{z}}{G_{z}+h \cdot \sin \theta}
$$

In that equation, $G_{x}$ and $G_{y}$ are the body's CG, $u$ is the horizontal displacement of the acting point of the floor reaction force, $F_{y}$ represents the horizontal component of the floor reaction force vector, $F_{z}$ represents the vertical component of the floor reaction force vector, $\theta$ is the angle from the acting point of the floor reaction force to the body's CG, $M$ is the rotational moment, and $r$ is the vertical distance from the body's CG to the line of the floor reaction force vector.

\section{Gait experiments}

We carried out gait measurement to obtain to measure the vertical component and the horizontal component of the floor reaction force vector using foot-pressure sensors. The systems used for measuring gait consist of a force plate sensor (9286; Kistler Instruments AG) and a motion capture system (Vicon140; Oxford Metrics Ltd.). Four infrared cameras were set on the $10 \mathrm{~m} \times 8 \mathrm{~m}$ floor. The walking corridor was $1.5 \mathrm{~m}$ wide and $4 \mathrm{~m}$ long, measuring three-dimensional images and the floor reaction force data at a sampling frequency of $60 \mathrm{~Hz}$. Our subject was a 20-year-old healthy man, $157 \mathrm{~cm}$ tall, weighing 54.8 $\mathrm{kg}$. We set gait cycles of 70, 90 and $110 \mathrm{step} / \mathrm{min}$.

\section{Gait results}

We estimated the vertical and horizontal components of the floor reaction force vector. We present an example in Fig. 3, which shows the experimental results for a gait cycle of 90 step/min. The solid line is the estimated value using the foot-pressure sensors; the dotted line is the measured value using the force plate. The measurement results from the force plate showed that the vertical component and the horizontal component floor reaction data were in good agreement with the estimate from the foot pressure sensor, starting from first contact by the heel through to the point where the toes leave the ground.

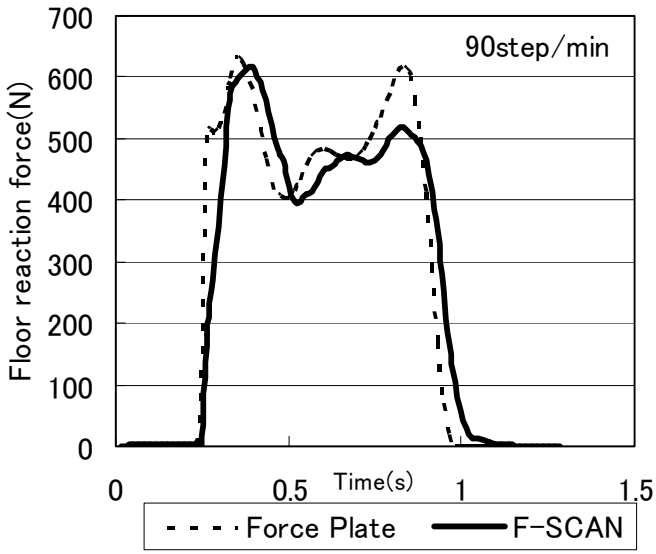

(a) Vertical component

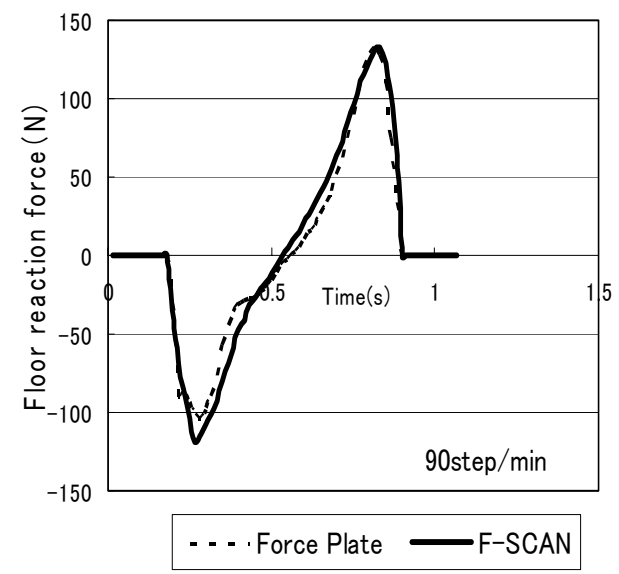

(b) Horizontal component

Fig. 3 Floor reaction force 


\section{Conclusions}

In this study, we proposed estimation of the vertical component and the horizontal component floor reaction force vector using wearable foot-pressure sensors for gait analysis. Future studies will examine the use of wearable sensor systems for a large range gait analysis system to replace the optical camera system with a magnetic sensor system.

\section{References}

(1) Obinata, G., Wearable Machines with the Times: Interaction between Human and Machines, Journal of JSME, Vol. 106, No. 1014 (2003), p. 30.

(2) Iwami, T, Miyawaki, K. and Obinata, G., Kinematical Analysis of Bi-Articular Muscles for Restoring Locomotion by FES, Transactions of the JSME, Series C, Vol. 65, No. 636 (1999), p. 3302-3308. 